\title{
POISSON BRACKETS AND COMMUTATOR BRACKETS. I
}

REESE T. PROSSER

\begin{abstract}
A previous result, that for certain problems in Hamiltonian mechanics the commutator bracket is determined by the Poisson bracket, is here extended to include certain problems in Hamiltonian field theory.
\end{abstract}

A fundamental component of any Hamiltonian formulation of mechanics, whether classical or quantum, is the Poisson bracket, which determines the development of the system in time. In an earlier paper [1] we have explored the close connection between a suitably defined Poisson bracket and the usual commutator bracket on the "moment algebra" generated by the canonical coordinates of a system of $n$ particles. There it was shown that under fairly natural assumptions the commutator bracket is necessarily a scalar multiple of the Poisson bracket; the scalar is zero in classical mechanics and is essentially Plank's constant in quantum mechanics.

In this paper we propose to extend this result so as to make it available for field theory. In order to do so, we must extend our previous assumptions in two ways: we must allow for countably many generators for our moment algebra, and we must allow for the anticommutators associated with Fermion fields. The first of these extensions is routine, but the second requires a little extra thought.

Let $A$ be the moment algebra of all (noncommutative) polynomials, with complex coefficients, in countably many generators $\left\{\varphi_{i}\right\}$, subject to a set of relations to be determined (cf. [1]).

We shall suppose, as in [1], that $A$ admits a conjugate-linear, antiisomorphic involution, called conjugation and denoted by $*$. We shall also suppose, in addition, that $A$ admits a linear isomorphic involution, called parity and denoted by $\pi$. Like the conjugation, the parity operation is determined by its action on the generators, where we assume

$$
\pi\left(\varphi_{i}\right)= \pm \varphi_{i}
$$

with the choice of sign to be specified later. To say that $A$ admits the involutions $*$ and $\pi$ is to say that $*$ and $\pi$ are compatible with whatever relations hold among the polynomials in $A$.

Received by the editors March 30, 1976.

AMS (MOS) subject classifications (1970). Primary 81A20.

O American Mathematical Society 1977 
The only eigenvalues of an involution are \pm 1 . If $f$ is an eigenpolynomial of $\pi$, we shall write

$$
\pi f=(-1)^{p} f
$$

where $p=p(f)=$ parity of $f$ is given by

$$
p(f)= \begin{cases}0 & \text { if } f \text { even } \\ 1 & \text { if } f \text { odd }\end{cases}
$$

Note that every polynomial $f \in A$ is a linear combination of eigenpolynomials

$$
f=g+h
$$

where

$$
g=\frac{1}{2}(f+\pi f), \quad h=\frac{1}{2}(f-\pi f) .
$$

We shall say that $A$ admits a (generalized) Poisson bracket if there is, compatible with the relations in $A$, a transformation

$$
\{,\}: A \times A \rightarrow A
$$

defined on $A \times A$ to $A$, which is bilinear,

$$
\begin{array}{r}
\{\lambda f+\mu g, h\}=\lambda\{f, h\}+\mu\{g, h\}, \\
\{f, \lambda h+\mu k\}=\lambda\{f, h\}+\mu\{f, k\},
\end{array}
$$

which is (generalized) skew symmetric,

$$
\{f, g\}=-(-1)^{p q}\{g, f\}
$$

where $f, g$ are eigenpolynomials of $\pi$ with parity $p, q$, and which is a (generalized) derivation,

$$
\begin{aligned}
& \{f, h k\}=\{f, h\} k+(-1)^{p r} h\{f, k\}, \\
& \{f g, h\}=(-1)^{q r}\{f, h\} g+f\{g, h\}
\end{aligned}
$$

where $f, g, h$ are eigenpolynomials of $\pi$ with parity $p, q, r$.

We note that (8) says that $\{f, g\}$ is skew-symmetric unless $f$ and $g$ are both of odd parity, in which case $\{f, g\}$ is symmetric. Similarly (9) says that $\{f, h k\}$ is a derivation of the product $h k$ unless $f$ and $h$ are both of odd parity, in which case $\{f, h k\}$ is a skew-derivation, satisfying the same law as does the exterior derivative acting on differential forms on a differential manifold.

We shall also require that this bracket be compatible with the involutions * and $\pi$ :

$$
\{f, g\}^{*}=\left\{g^{*}, f^{*}\right\}, \quad \pi\{f, g\}=\{\pi f, \pi g\}
$$


Properties (7)-(10) determine the Poisson bracket completely once it is known on the generators. For these we specify

$$
\left\{\varphi_{i}, \varphi_{j}\right\}=\lambda_{i j} 1
$$

where the $\lambda_{i j}$ are (complex) scalars, not all zero.

Next we define on $A$ the (generalized) commutator

$$
[,]: A \times A \rightarrow A
$$

which is bilinear, and which satisfies

$$
[f, g]=f g-(-1)^{p q} g f
$$

where $f, g$ are eigenpolynomials of $\pi$ with parity $p, q$. Property (12) says that $[f, g]$ is the ordinary commutator $f g-g f$ unless $f$ and $g$ are both of odd parity, in which case $[f, g]$ is the anticommutator $f g+g f$. It is a straightforward exercise to verify that the generalized commutator also satisfies properties (8)-(10), and hence provides an example of a generalized Poisson bracket for $A$ whenever it satisfies property (10a). This is precisely the case if $A$ is the moment algebra generated by a Boson field $\left(\left[\varphi_{i}, \varphi_{j}\right]_{-}=i \delta_{i j} 1\right)$ or a Fermion field $\left(\left[\varphi_{i}, \varphi_{j}\right]_{+}=\delta_{i j} 1\right)$.

Our principal result here is that the generalized commutator is essentially the only possible example of a generalized Poisson bracket.

THEOREM. If $A$ admits a generalized Poisson bracket $\{$,$\} , then the generalized$ commutator bracket [, ] is given by

$$
[,]=z\{,\}
$$

where the polynomial $z$ lies in the center of $A$.

The proof is an immediate consequence of the following result:

Lemma. If $f, g, h, k \in A$, then

$$
\{f, h\}[g, k]=[f, h]\{g, k\} .
$$

Proof. We assume that $f, g, h, k$ have parity $p, q, r, s$, and expand the Poisson bracket $\{f g, h k\}$ in two ways, first using $(9 \mathrm{a})$ and then $(9 \mathrm{~b})$, and second using (9b) and then (9a):

$$
\begin{aligned}
\{f g, h k\}= & \{f g, h\} k+(-1)^{(p+q) r} h\{f g, k\}=(-1)^{q r}\{f, h\} g k+f\{g, h\} k \\
& +(-1)^{p r+q r+q s} h\{f, k\} g+(-1)^{p r+q r} h f\{g, k\}, \\
\{f g, h k\}= & (-1)^{q(r+s)}\{f, h k\} g+f\{g, h k\} \\
= & (-1)^{q r+q s}\{f, h\} k g+(-1)^{q r+q s+p r} h\{f, k\} g \\
& +f\{g, h\} k+(-1)^{q r} f h\{g, k\} .
\end{aligned}
$$

Subtracting (16) from (15), we obtain 


$$
0=(-1)^{q r}\left(\{f, h\}\left(g k-(-1)^{q s} k g\right)-\left(f h-(-1)^{p r} h f\right)\{g, k\}\right) .
$$

Now (17), together with (12), yields (14) for eigenpolynomials of $\pi$. The general case follows from the bilinearity of both brackets and (4).

To prove the Theorem, we have simply to take $f$ and $h$ arbitrary in $A$, and $g$ and $k$ so that $\{g, k\}=1$. This last can always be achieved by taking $g$ and $k$ as scalar multiples of generators, by (10a), so long as the $\lambda_{i j}$ are not all zero. Then we put $z=[g, k]$, and substitute in (14):

$$
\{f, h\} z=[f, h] 1 .
$$

To show that $z$ does not depend on the choice of $g$ and $k$, suppose $\left\{g^{\prime}, k^{\prime}\right\}=1$ also, and put $\left[g^{\prime}, k^{\prime}\right]=z^{\prime}$. Now substitute $f=g^{\prime}, h=k^{\prime}$ in (18) to see that $z=z^{\prime}$. To show that $z$ lies in the center of $A$, let $f$ be any generator of $A$, and consider, with $p, q$ the parities of $f, g$,

$$
\{f, z\}=\{f,[g, k]\}=[\{f, g\}, k]+(-1)^{p q}[g,\{f, k\}]=0,
$$

since $\{f, g\}$ and $\{f, k\}$ are scalar multiples of the identity. But if $\{f, z\}=0$, then by (18)

$$
[f, z]=\{f, z\} z=0 .
$$

Now $z$ is of even party. To see this, note that

$$
\pi z=\pi[g, k]=[\pi g, \pi k]=(-1)^{q+s}[g, k]=\left(-1^{q+s} z\right) .
$$

Similarly,

$$
\pi\{g, k\}=(-1)^{q+s}\{g, k\} .
$$

But $\{g, k\}=1$ has even parity, so $(-1)^{q+s}=+1$, and so by (22) $z$ has even parity. Hence

$$
[f, z]=f z-z f=0
$$

by (21), and so $z$ commutes with each generator $f$ of $A$, and so lies in the center of $A$. The proof is complete.

We distinguish several special cases. If the generators all have even parity, then so does every polynomial in $A$, and $\pi$ reduces to the identity. In this case, the brackets are both skew-symmetric and the generalized commutator reduces to the ordinary commutator. In this case, if $z=0$ then all commutators vanish and the algebra $A$ is the commutative algebra on countably many generators, with no other relations (cf. [1]). This is the case determined by classical mechanics of commuting fields. If $z=i \hbar 1, \hbar$ a real scalar, then relations (10) and (13) yield the Heisenberg commutation relations, and the algebra $A$ is the universal enveloping algebra of the Heisenberg Lie algebra determined by these relations. This is the case determined by the quantum mechanics of Boson fields. If $z$ is any other polynomial it must be Hermitian 
and square-free, and the algebra so determined is a suitable combination of those described above (cf. [1]).

If the generators all have odd parity, then so does every monomial of odd degree in $A$, and the brackets are both symmetric on monomials of odd degree. In particular, the generalized commutator bracket reduces to the anticommutator on the generators. In this case, if $z=0$, the anticommutators of the generators all vanish, and the algebra $A$ becomes the Grassmann algebra on countably many generators, with no other relations. This case cannot serve as a model for any mechanics because the square of each generator vanishes, and it follows that the expectation value of each generator vanishes in every state (cf. [1]). This explains why there is no useful classical analogue of the Fermion fields. If $z=\hbar 1$, then relations (10) and (13) yield the Fermion anticommutation relations, and the algebra $A$ is the Clifford algebra determined by these relations. This is the case determined by the quantum mechanics of Fermion fields. If $z$ is any other polynomial it must be Hermitian and square-free, and the algebra so determined is again a suitable combination of those described above. We shall not pursue the details here.

Finally if some generators are of odd parity and some even, then the even generators commute with the odd ones, since if $\varphi_{i}$ is even and $\varphi_{j}$ odd, then

$$
\lambda_{i j} 1=\pi\left(\lambda_{i j} 1\right)=\pi\left\{\varphi_{i}, \varphi_{j}\right\}=\left\{\pi \varphi_{i}, \pi \varphi_{j}\right\}=-\left\{\varphi_{i}, \varphi_{j}\right\}=-\lambda_{i j} 1
$$

It follows that $\left\{\varphi_{i}, \varphi_{j}\right\}=0$, and hence by $(18)$ that $\left[\varphi_{i}, \varphi_{j}\right]=0$. But if $\varphi_{i}$ is even and $\varphi_{j}$ is odd, then $\left[\varphi_{i}, \varphi_{j}\right]=\varphi_{i} \varphi_{j}-\varphi_{j} \varphi_{i}=0$. It follows that $A$ is the tensor product of two subalgebras, one of Boson type generated by the even generators, and one of Fermion type generated by the odd generators, and each of those subalgebras has the structure described above.

We see now that the signs in (1) are determined by the rule

$$
\pi \varphi_{i}= \begin{cases}+\varphi_{i} & \text { if } \varphi_{i} \text { is a Boson field element } \\ -\varphi_{i} & \text { if } \varphi_{i} \text { is a Fermion field element }\end{cases}
$$

\section{BIBLIOGRAPHY}

1. R. T. Prosser, A new formulation of particle mechanics, Mem. Amer. Math. Soc. No. 61 (1967), 57 pp. MR 32 \#8545.

2., A brief derivation of the Heisenberg commutation relations, Proc. Amer. Math. Soc. 26 (1970), 640-641. MR 44 \#7853.

Department of Mathematics, Dartmouth College, Hanover, New Hampshire 03755 